\title{
Monozygotic twins with CAPN5 autosomal dominant neovascular inflammatory vitreoretinopathy
}

This article was published in the following Dove Press journal:

Clinical Ophthalmology

5 December 2012

Number of times this article has been viewed

\author{
Hannah A Rowell 1,2 \\ Alexander G Bassuk ${ }^{3,4}$ \\ Vinit B Mahajan ${ }^{1,2}$ \\ 'Omics Laboratory, ${ }^{2}$ Department \\ of Ophthalmology and Visual \\ Sciences, ${ }^{3}$ Department of Pediatrics, \\ ${ }^{4}$ Department of Neurology, University \\ of lowa, lowa City, IA, USA
}

Background: The purpose of this study was to describe the clinical findings in a set of monozygotic twins with autosomal dominant neovascular inflammatory vitreoretinopathy (ADNIV) over a 23-year period.

Methods: A pair of female twins were examined between 26 and 49 years of age. The concordance and discordance of their clinical features were determined. The CAPN5 gene was sequenced using genomic DNA.

Results: Both twins of an affected father demonstrated Stage I ADNIV with mild vitreous cells and a negative b-wave on electroretinography. Genetic analysis confirmed a guanine to thymine nucleotide (c.728G $>$ T, pArg243Leu) mutation in the CAPN5 gene. Over the course of 23 years, each twin progressed to stage III disease, showing posterior uveitis, cystoid macular edema, intraocular fibrosis, early retinal neovascularization, retinal degeneration, and cataract. Disease progression varied moderately between each twin and was asymmetrical between eyes. Twin A had 20/70 and 20/125 in the right and left eye, respectively, and underwent vitrectomy surgery and intravitreal injections with bevacizumab for recurrent cystoid macular edema. Twin B maintained 20/20 and 20/40 in the right and left eye, respectively without intervention.

Conclusion: There was asymmetry between the eyes and some discordance in the rate of disease progression in these monozygotic twins with ADNIV. The overall high disease concordance suggests genetic factors play a major role in clinical manifestations in CAPN5 vitreoretinopathy. Keywords: autosomal dominant neovascular inflammatory vitreoretinopathy, ADNIV, CAPN5, calpain-5, monozygotic twins

\section{Introduction}

Autosomal dominant neovascular inflammatory vitreoretinopathy (ADNIV) is an inherited autoimmune uveitis and vitreoretinal degeneration. ${ }^{1-3}$ ADNIV can be divided into five stages, each lasting approximately ten years. ${ }^{4}$ Patients present during the second or third decade of life with posterior uveitis and reduction of the electroretinogram b-wave. They become more symptomatic when cataracts, cystoid macular edema, and disc edema diminish visual acuity during the second stage. Severe vision loss begins during the third stage when proliferative retinal neovascularization and epiretinal membranes appear. There is an ongoing pigmentary retinal degeneration and peripheral visual field loss during all stages. In the fourth stage, proliferative vitreoretinopathy causes tractional retinal detachments at the macula and vitreous base. The fifth or end-stage disease is marked by phthisis. Cell-mediated autoimmunity may be an underlying disease mechanism. ${ }^{5}$

ADNIV is caused by mutations in the CAPN5 gene located on chromosome $11 \mathrm{q} 13 .{ }^{1}$ CAPN5 encodes the protease, calpain-5, a calcium-activated cysteine protease. ${ }^{1}$ 
In two large ADNIV pedigrees, we identified nonsynonymous missense mutations. A CAPN5 mutation c. $728 \mathrm{G}>\mathrm{T}$, pArg243Leu was found in the first family and a nearby c.731T $>$ C, pLeu244Pro was identified in the second family. ${ }^{1}$ To gain insight into the phenotypic expressivity of CAPN5 mutations, we describe the clinical findings in monozygotic female twins from the first ADNIV family that were followed over 23 years.

\section{Materials and methods}

The study was approved by the Institutional Review Board for Human Subjects Research at the University of Iowa, was compliant with the Health Insurance Portability and Accountability Act, and adhered to the tenets of the Declaration of Helsinki. A retrospective case series was assembled from the charts of two ADNIV patients from The University of Iowa. Genetic testing was performed as previously described. ${ }^{1}$ Stereoscopic color fundus images were obtained using a Topcon TRC 50DX camera (Topcon, Pyramus, NJ). The subjects deferred fluorescein angiography studies. Optical coherence tomography imaging was obtained from the spectral-domain Heidelberg HRA2 Spectralis, version 1.6.1 (Heidelberg Engineering Inc, Vista, CA). A full-field electroretinogram was performed using DTL recording electrodes and Ganzfeld stimulation according to international standards, as previously described. ${ }^{3}$ Briefly, the eyes were dilated and dark adapted for 30 minutes. Electroretinograms were recorded simultaneously from both eyes using Burian-Allen bipolar contact lens electrodes. Evoked waveforms, a $100 \mu \mathrm{V}$ calibration pulse, and a stimulus artifact were recorded on Polaroid film. ${ }^{3}$ Both sisters deferred electroretinography at the most recent examination.

\section{Results}

In two ADNIV pedigrees with 150 and 79 respective family members, there has been only one set of monozygotic twins. In the first ADNIV pedigree, twin sisters had an affected father (Figure 1A). Genetic testing verified the CAPN5 guanine to thymine nucleotide change (c.728G $>\mathrm{T}$, pArg243Leu) in all affected family members. ${ }^{1}$

Both twins were asymptomatic when first evaluated at 26 years of age and had no health issues. Twin A had a visual acuity of 20/20 in both eyes (OU) with a hyperopic refraction of $-0.25+1.25 \times 85$ in the right eye $(\mathrm{OD})$ and $-0.50+1.50 \times 90$ in the left eye (OS). Twin B had a similar visual acuity of $20 / 15$ OU with a hyperopic refraction of $-0.50+1.00 \times 90 \mathrm{OD}$ and $-0.25+1.00 \times 88 \mathrm{OS}$. In both cases, the visual fields were full to confrontation, ocular motility was normal, there was no relative afferent pupillary defect, and intraocular pressures were $15-16 \mathrm{mmHg}$ OU. The anterior segment examinations were unremarkable, except for a small pupillary membrane OS in twin A. There were no anterior chamber cells, flare, or cataract. Dilated fundus examinations showed trace vitreous cells but no disc or retinal neovascularization, cystoid macular edema, or epiretinal membranes. In the retinal periphery of twin A, there were cystoid changes, a few sheathed vessels, yellow spots, but no bone-spicule pigmentation or pigment clumping, and the periphery was well vascularized (Figure 1B). In the retinal periphery of twin $\mathrm{B}$, there was mild pigment granularity, cystoid changes, and small areas suggesting incomplete vascularization (Figure 1C). Electroretinography in twin A showed that a photopic b-wave was approximately $70 \%$ of normal and a scotopic b-wave was $68.5 \%$ of normal (Figure 1D and E). Twin B showed that a photopic b-wave was approximately $32.5 \%$ of normal and a scotopic b-wave was $83 \%$ of normal (Figure 1D and E). These findings, along with their family history, were consistent with stage I ADNIV (Figure 5).

Both twins were examined one year later, and their examination was stable, except for the appearance of $1+$ vitreous cells. Five years later, the visual acuity remained the same, but there were $2+$ vitreous cells and peripheral pigmentary changes consistent with Stage II ADNIV.

Twenty-three years after their initial examination, the ADNIV twins were examined at the age of 49 years. There were no significant health differences. They had progressed to stage III ADNIV. However, the findings in twin A were more severe than in twin $\mathrm{B}$. The best-corrected visual acuity of twin A had deteriorated to 20/70 OD and 20/125 OS with a hyperopic refraction of $+1.25+1.50 \times 105$ OD and $-0.50+2.50 \times 93 \mathrm{OS}$. The reduction in visual acuity in twin B was less severe at 20/40 OD and 20/20 OU with a hyperopic refraction of $+1.00+1.75 \times 92$ OD and $+0.75+2.50 \times 89$ OS. Twin A showed a $2+$ nuclear sclerotic and $1+$ posterior subcapsular cataract OD, and she had previously undergone cataract surgery with posterior chamber lens implantation OS. A dilated fundus examination of the right eye in twin A showed a 1+ epiretinal membrane, cystoid macular edema, peripheral pigmentary changes, and asteroid. The left eye showed cystoid macular edema, an obliterated vessel in the peripheral macula, and some early vascular remodeling (Figure 2A). Twin A had previously undergone a vitrectomy and internal limiting membrane peel for an epiretinal membrane with cystoid 
A

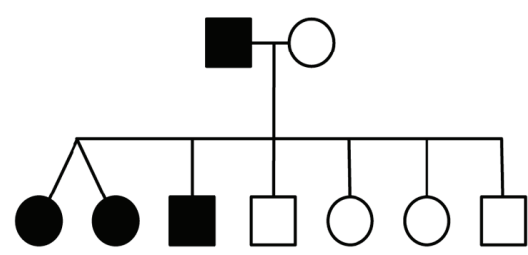

Twin A Twin B

B

Twin A

OD

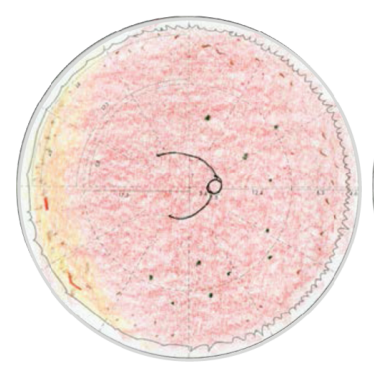

D
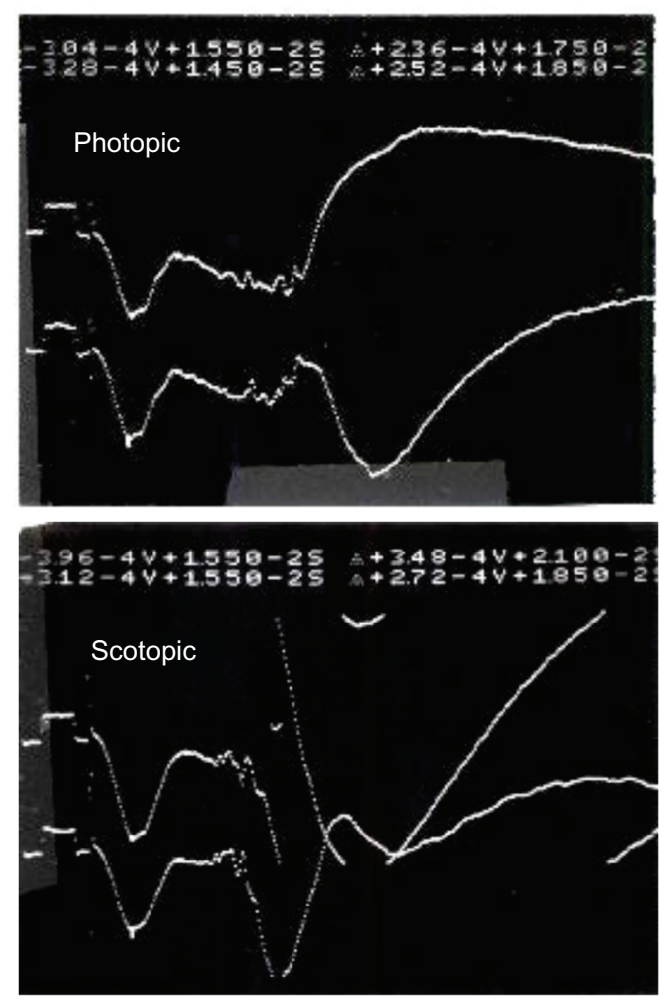

OS

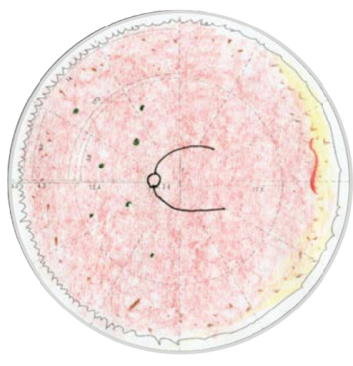

Twin A
C

E

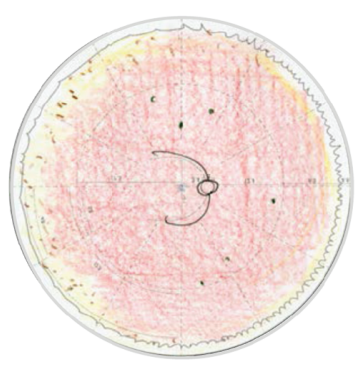

Twin B

OD

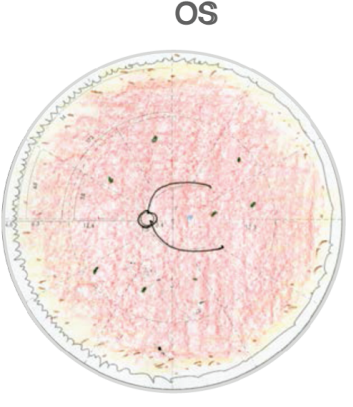

Twin B
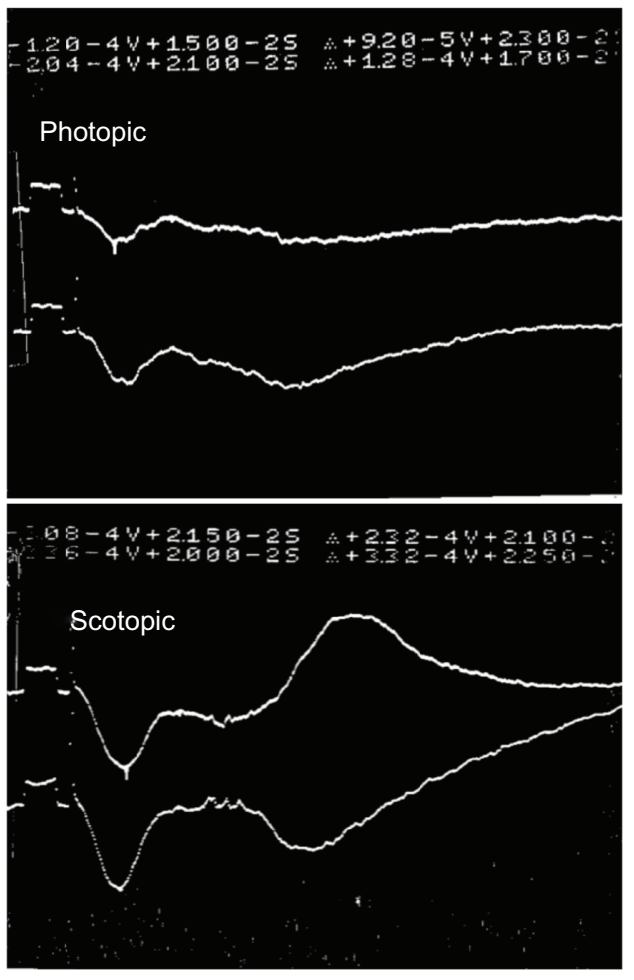

Figure I Monozygotic twin sisters display stage I ADNIV at the age of 26 years. (A) Pedigree of female monozygotic twins who inherited the CAPN5 mutation (c.728G >T, pArg243Leu) from their father in the first ADNIV family. Black symbols represent clinically affected subjects. Open symbols represent unaffected subjects. (B) Fundus drawings of twin A recorded peripheral cystoid changes, a few sheathed vessels, and yellow spots. (C) Fundus drawings of twin B recorded peripheral pigment granularity, cystoid changes, and small areas suggesting incomplete vascularization. (D and $\mathbf{E}$ ) Electroretinographic tracings from twins $A$ and $B$ show reduction of $b$-wave relative to the a-wave. Photopic electroretinogram (top panel) and scotopic electroretinogram (bottom panel).

Notes: The normal photopic b-wave amplitude using this system was $339 \pm 85$ microvolts and the normal scotopic b-wave was $450 \pm 100$ microvolts. The calibration mark (initial spike) before stimulus shows 100 microvolts.

Abbreviation: ADNIV, autosomal dominant neovascular inflammatory vitreoretinopathy. 
A

OD
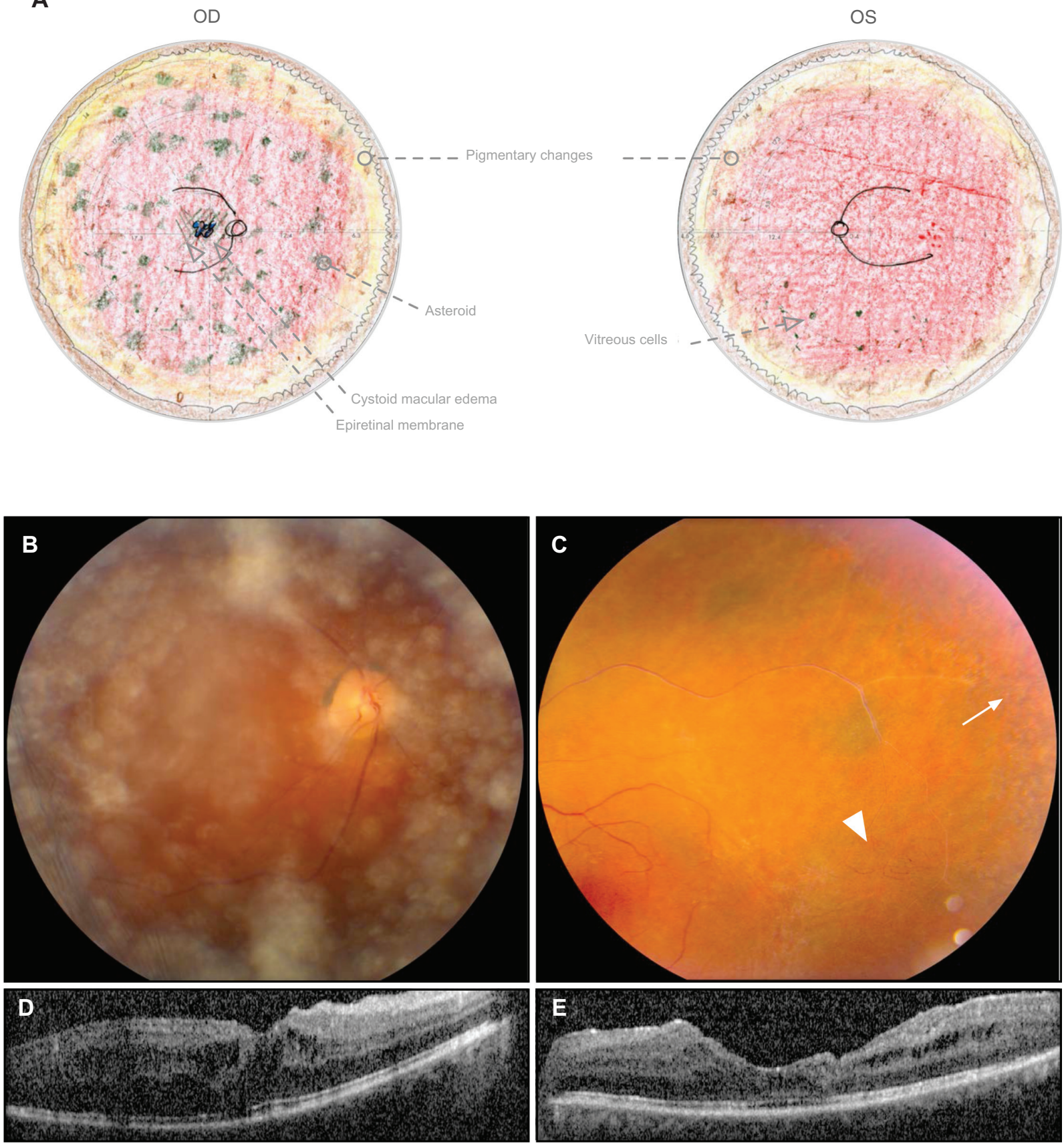

Figure 2 Twin A displays stage III ADNIV at the age of 49 years. (A) Fundus drawings record I+ epiretinal membrane, asteroid, and cystoid macular edema OD; attenuated vessels and vascular remodeling OS; and peripheral pigmentary changes and mild vitreous cells OU. (B) Fundus photograph OD shows asteroid in the vitreous. (C) Fundus photograph OS shows a sheathed vessel, pigmentary changes (arrow) in the peripheral macula, and vascular remodeling (arrowhead). (D and E) Optical coherence tomography of the foveas shows cystoid macular edema.

Abbreviations: ADNIV, autosomal dominant neovascular inflammatory vitreoretinopathy; OD, right eye; OS, left eye; OU, both eyes.

macular edema OS. She had also received two bevacizumab injections for recurrent cystoid macular edema OD, but had shown a very limited response.

Like her sister, twin B had also developed epiretinal membranes, but the cystoid macular edema was less severe and not treated. In the right eye, there was fibrotic vitreous traction at the disc. There was also a new patch of dark pigmentation nasal to the disc (Figure 3B). This may represent retinal pigment epithelial changes from traction rather than retinal degeneration. There were dilated retinal capillaries and early neovascularization temporal to the macula. Vitreous bands were present. In the peripheral retina, there was atrophy, patchy pigmentation, and fibrosis at the vitreous base without neovascularization. Fundus 
A

OD
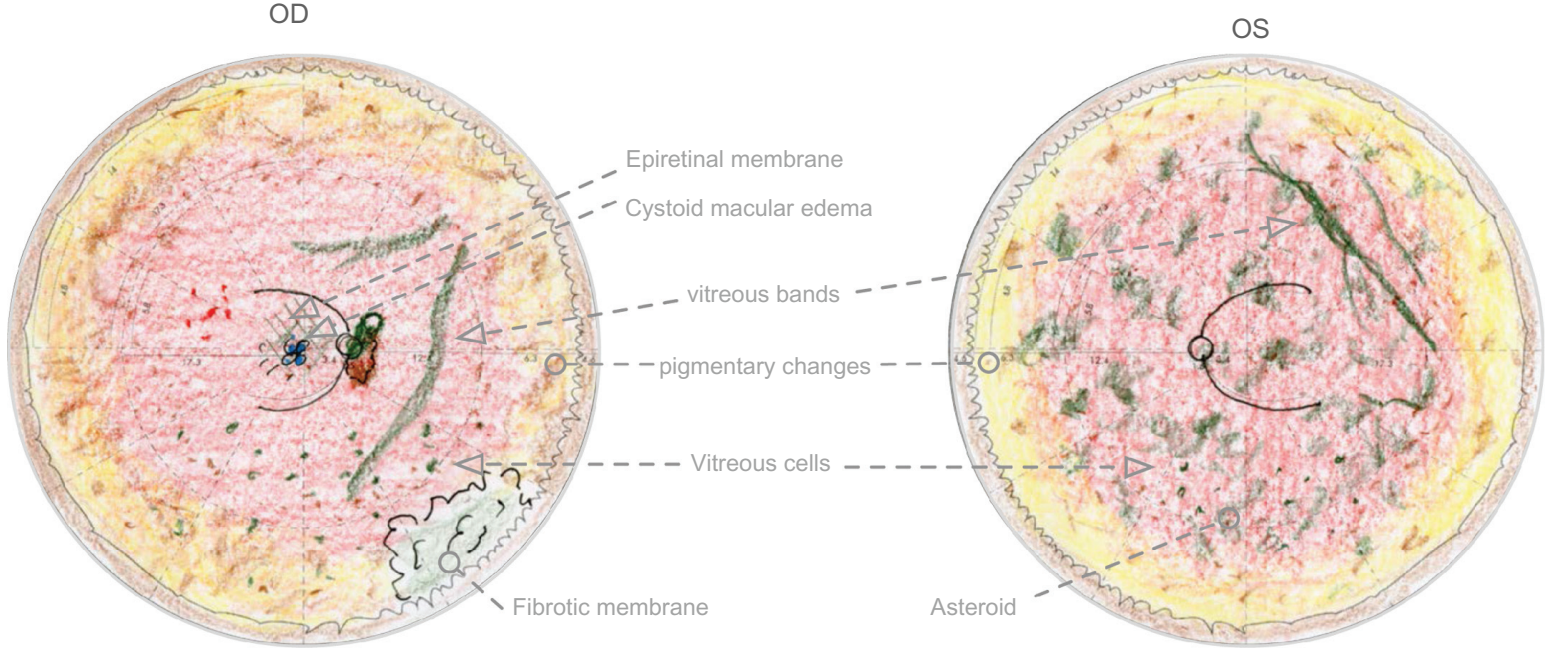
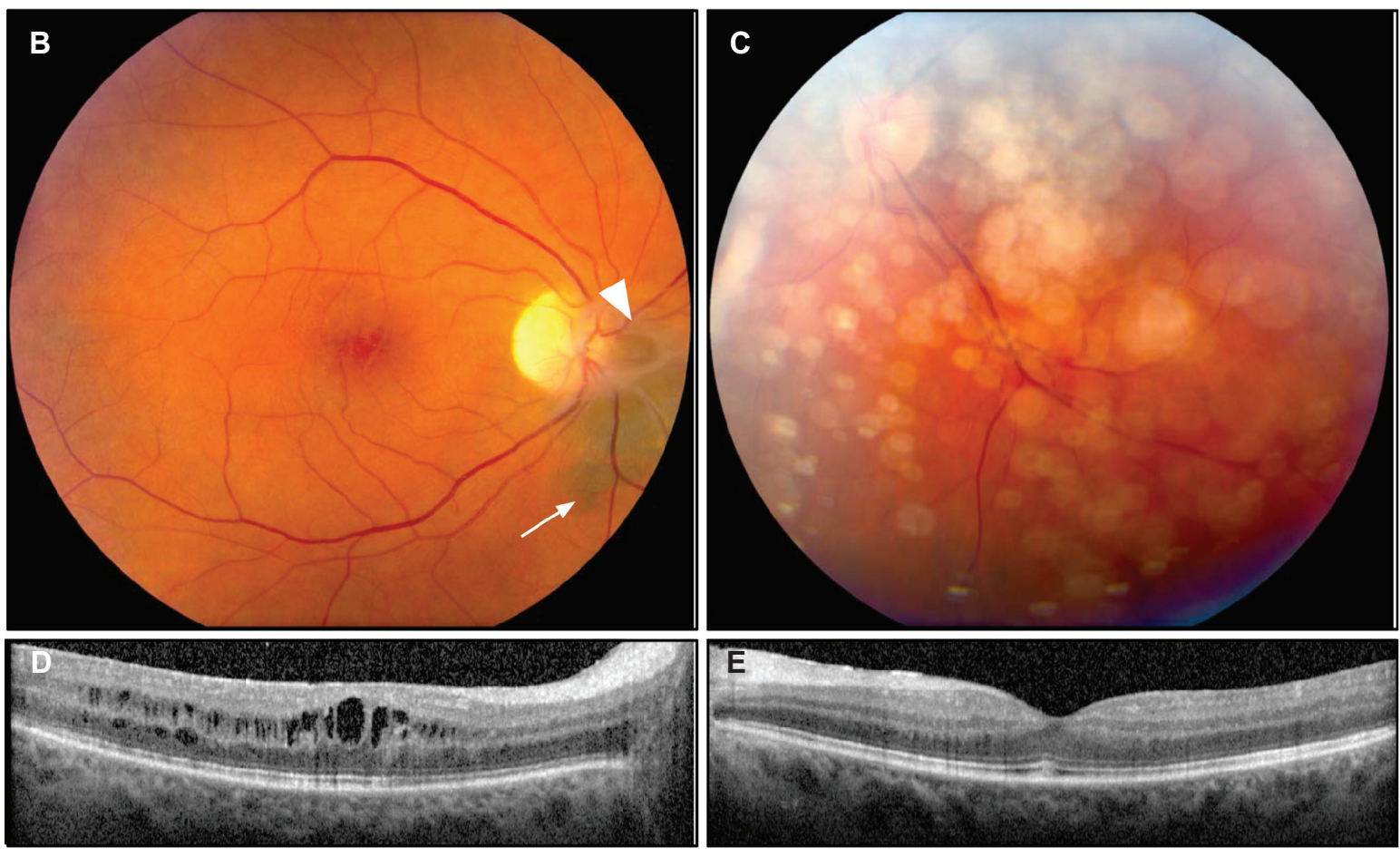

Figure 3 Twin B displays stage III ADNIV at the age of 49 years. (A) Fundus drawings record less severe epiretinal membrane and cystoid macular edema only OD. There was fibrotic vitreous traction at the disc and a fibrotic membrane over the pars plana OD. The peripheral retina showed pigmentary and atrophic changes. There were vitreous cells and vitreous bands OU. (B) Fundus photograph OD shows an epiretinal membrane and cystoid macular edema. At the optic disc there was fibrous traction (arrowhead) and pigmentary changes (arrow). (C) Fundus photograph OS shows asteroid in the vitreous. (D) Optical coherence tomography OD shows cystoid macular edema. (E) There was no cystoid macular edema OS.

Abbreviations: ADNIV, autosomal dominant neovascular inflammatory vitreoretinopathy; OD, right eye; OS, left eye; OU, both eyes.

examination of her left eye showed vitreous bands, asteroid, and pigmentary changes in the macula (Figure 3A). Both eyes showed mild posterior subcapsular cataracts. At this time, there were moderate vitreous cells and some vitreous bands OU.

Goldmann visual field testing showed constriction of the peripheral fields in both twins, especially inferonasally.
The constriction was more severe in twin A, who had central scotomas corresponding to cystoid macular edema and an approximate 10-degree greater constriction of the I2e, I4e, and V4e isopters OU (Figure 4). Twin B showed a scotoma in the right eye and enlargement of the blind spot, corresponding to the fibrotic membrane and pigmentary changes near the optic nerve head. 

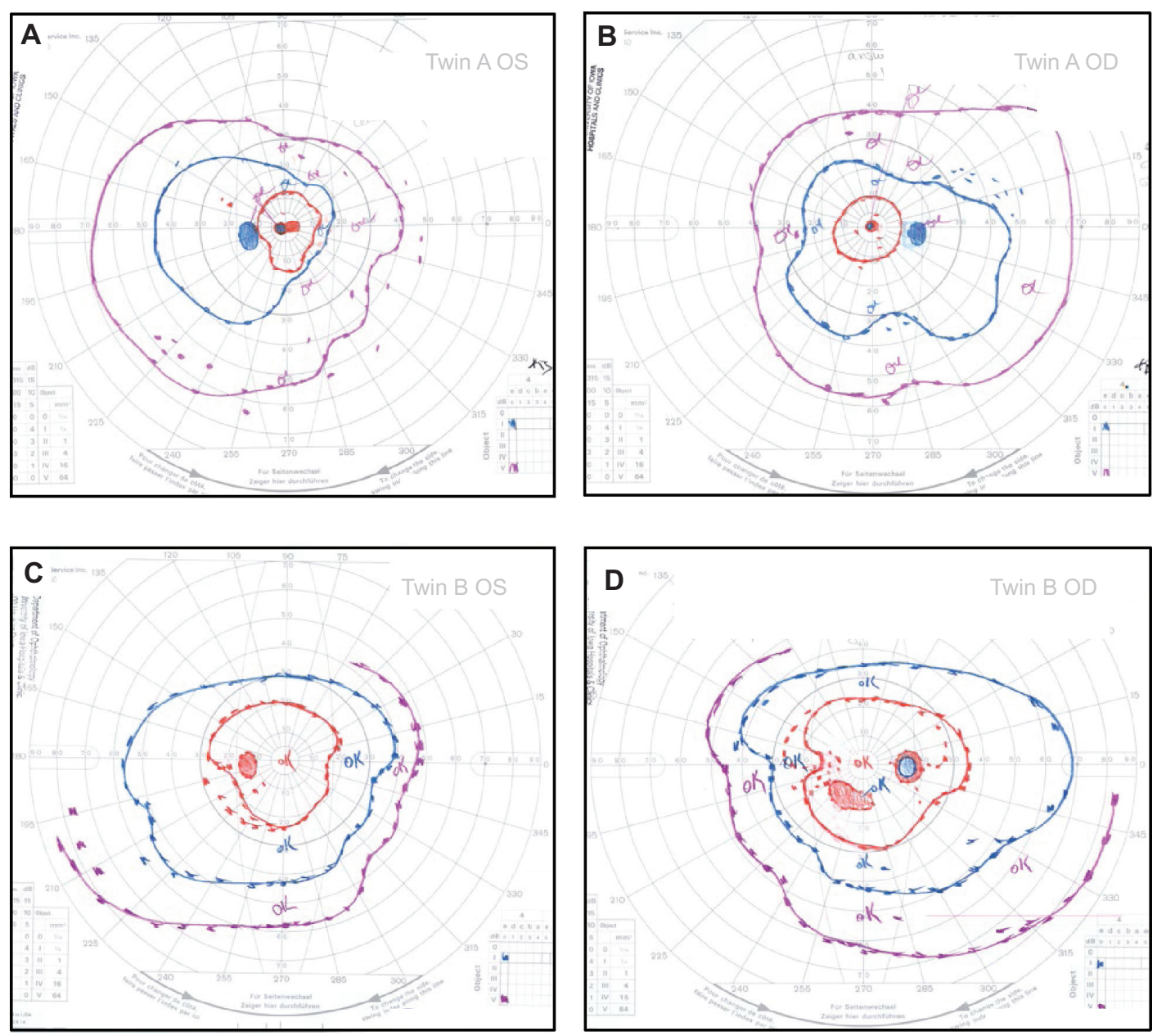

Figure 4 Goldmann visual fields at the age of 49 years. (A and B) Twin A shows central scotomas and more severe constriction of all isopters by approximately 10 degrees compared with twin B. (C and D) Twin B shows less severe peripheral constriction of isopters and a scotoma OD. (I2e, red; 14e, blue; V4e, purple).

Abbreviations: OD, right eye; OS, left eye.

\section{Discussion}

CAPN5 represents the first nonsyndromic gene to cause uveitis and is among the very few genes known to cause autoimmunity in a Mendelian fashion. ${ }^{1,6}$ ADNIV patients do not develop any systemic autoimmune conditions. The cellular infiltrates seem to involve only the eye, ${ }^{5}$ and cellmediated autoimmunity may be a key mechanism. ${ }^{5}$ CAPN5 mutations also lead to other blinding pathologies that include photoreceptor degeneration, intraocular fibrosis, and neovascularization. Calpains activate various signaling pathways, and an unregulated gain of function might explain the complex phenotype. ${ }^{1}$

In this report, the phenotype of monozygotic twins with ADNIV was ascertained over 23 years. When they were initially examined, each presented with nearly identical examination findings. Both were asymptomatic with excellent visual acuity, but there were vitreous cells and an abnormal electroretinogram. The photopic electroretinogram was worse in twin B. Over the course of their disease, there was general concordance with respect to the stage of disease, but there was discordance with the rate of progression and severity of examination features. While grading of vitreous cells was equal in both eyes, features such as cystoid macular edema, neovascularization, and fibrosis varied between the twins. The major disease feature causing vision loss in twin A was cystoid macular edema, which was caused by chronic inflammation and epiretinal membranes. Interestingly, there was also asymmetry between left and right eyes. The difference between left and right is consistent with our observations in other patients with ADNIV. Several of the ADNIV patients, for example, have developed blindness in one eye before the other, remaining partially sighted for years.

Analysis of twin phenotypes can give insight into the relative contribution of genetic and environmental factors to ophthalmic disease. ${ }^{7}$ Phenotypic discordance among twins may be due to epigenetic, postzygotic, or environmental differences, and this has been observed in other retinal diseases. Twins with retinitis pigmentosa, a progressive 
A

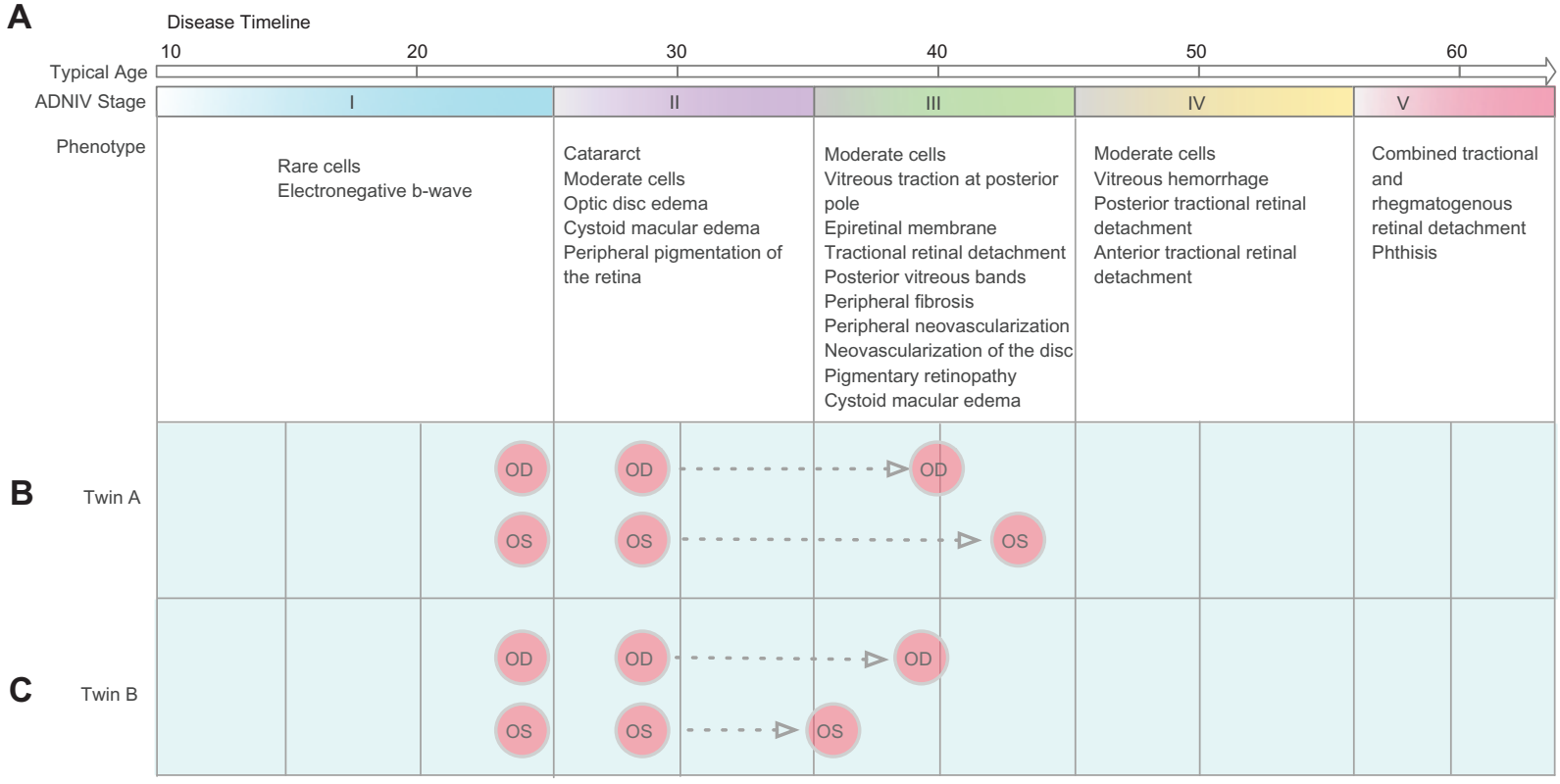

Figure 5 ADNIV disease timeline and progression. (A) ADNIV occurs in five stages (I-V), each lasting approximately 10 years and beginning at I0-30 years of age. Clinical features are listed below each stage. (B) Twin A progressed to more severe stage III disease with some asymmetry between eyes. Her epiretinal membranes and cystoid macular edema were worse in the left eye and required surgery. There was also more peripheral field loss. (C) Twin B progressed to less severe stage III disease with asymmetry between eyes. Cystoid macular edema was worse in the right eye.

Abbreviations: ADNIV, autosomal dominant neovascular inflammatory vitreoretinopathy; OD, right eye; OS, left eye.

degenerative disease of the retina, can show discordance..$^{8-11}$ There are known cases of monozygotic twin pairs with identical gene mutations where one sibling displayed retinitis pigmentosa and the other appeared unaffected. ${ }^{11,12}$ In other studies, twins with age-related macular degeneration, exhibited concordant features, such as drusen distribution characteristics. ${ }^{13,14}$ Developmental factors, such as methylation, can modulate gene expression, which may be relevant in autoimmunity. ${ }^{6}$ ADNIV is an autoimmune disease, and monozygotic twins with immunological disease can show greater concordance than dizygotic twins when comparing the methylation status of their DNA. ${ }^{6}$ The ADNIV twins here are mostly concordant with respect to their stage of disease, but show discordance in specific features, disease severity, and vision loss. Both ADNIV sisters grew up in the same household as children and in the same city, and did not have other work or health differences over the 23-year follow-up, suggesting that environmental factors were not significantly different. Limitations of twin studies, including this report, are the small population sizes and inability to account for possible postzygotic genetic and epigenetic events. $^{12}$

\section{Conclusion}

Our findings in these twins emphasize that ADNIV patients need careful, continuous long-term follow-up to address ongoing chronic inflammation, membrane formation, neovascularization, and retinal degeneration. ${ }^{3}$ Genetic factors are important, but there can be differences in the rate and progression between eyes, and closely related family members may require different timing for therapeutic intervention. ${ }^{4}$

\section{Acknowledgments}

The authors are supported by The University of Iowa Medical Student Research Summer Fellowship (HAR), and National Institutes of Health (K08EY020530) and Research to Prevent Blindness (VBM). The authors gratefully acknowledge James C Folk MD and Jared Nielsen MD who provided care for the patients, and Edwin M Stone MD, PhD for DNA analysis.

\section{Disclosure}

The authors have no commercial or financial interests associated with this work.

\section{References}

1. Mahajan VB, Skeie JM, Bassuk AG, et al. Calpain-5 mutations cause autoimmune uveitis, retinal neovascularization, and photoreceptor degeneration. PLoS Genet. 2012;8:e1003001.

2. Stone EM, Kimura AE, Folk JC, et al. Genetic linkage of autosomal dominant neovascular inflammatory vitreoretinopathy to chromosome 11q13. Hum Mol Genet. 1992;1:685-689.

3. Bennett SR, Folk JC, Kimura AE, Russell SR, Stone EM, Raphtis EM. Autosomal dominant neovascular inflammatory vitreoretinopathy. Ophthalmology. 1990;97:1125-1135. 
4. Tlucek PS, Folk JC, Orien JA, Stone EM, Mahajan VB. Inhibition of neovascularization but not fibrosis with the fluocinolone acetonide implant in autosomal dominant neovascular inflammatory vitreoretinopathy. Arch Ophthalmol. July 9, 2012. [Epub ahead of print.]

5. Mahajan VB, Vallone JG, Lin JH, et al. T-cell infiltration in autosomal dominant neovascular inflammatory vitreoretinopathy. Mol Vis. 2010; 16:1034-1040.

6. Zenewicz LA, Abraham C, Flavell RA, Cho JH. Unraveling the genetics of autoimmunity. Cell. 2010;140:791-797.

7. Seddon JM, Cote J, Page WF, Aggen SH, Neale MC. The US twin study of age-related macular degeneration: relative roles of genetic and environmental influences. Arch Ophthalmol. 2005;123:321-327.

8. Attarzadeh A, Hoseinirad A, Rahat F. Brown syndrome in one pair of dizygotic twins: a case report. Cases J. 2010;3:1.

9. Machida S, Takahashi T, Gotoh N, Yoshimura N, Fujiwara T, Kurosaka D. Monozygotic twins with polypoidal choroidal vasuculopathy. Clin Ophthalmol. 2010;4:793-800.
10. Tuft SJ, Hassan H, George S, Frazer DG, Willoughby CE, Liskova P. Keratoconus in 18 pairs of twins. Acta Ophthalmol. 2012;90: e482-e486.

11. Walia S, Fishman GA, Swaroop A, et al. Discordant phenotypes in fraternal twins having an identical mutation in exon ORF15 of the RPGR gene. Arch Ophthalmol. 2008;126:379-384.

12. Berghmans LV, de Mendonca RH, Coppieters F, et al. Discordance for retinitis pigmentosa in two monozygotic twin pairs. Retina. 2011;31: 1164-1169.

13. Quellec G, Russell SR, Seddon JM, et al. Automated discovery and quantification of image-based complex phenotypes: a twin study of drusen phenotypes in age-related macular degeneration. Invest Ophthalmol Vis Sci. 2011;52:9195-9206.

14. Klein ML, Mauldin WM, Stoumbos VD. Heredity and age-related macular degeneration. Observations in monozygotic twins. Arch Ophthalmol. 1994;112:932-937.
Clinical Ophthalmology

\section{Publish your work in this journal}

Clinical Ophthalmology is an international, peer-reviewed journal covering all subspecialties within ophthalmology. Key topics include: Optometry; Visual science; Pharmacology and drug therapy in eye diseases; Basic Sciences; Primary and Secondary eye care; Patient Safety and Quality of Care Improvements. This journal is indexed on

\section{Dovepress}

PubMed Central and CAS, and is the official journal of The Society of Clinical Ophthalmology (SCO). The manuscript management system is completely online and includes a very quick and fair peer-review system, which is all easy to use. Visit http://www.dovepress.com/ testimonials.php to read real quotes from published authors. 